\title{
Developing a Diabetes Smartphone App
}

\author{
Dr Serife Mehmet
}

Consultant Physician in Diabetes \& Endocrinology, Lewisham \& Greenwich NHS Trust, Diabetes Centre, Queen Mary's Hospital, Frognal Avenue, London, DA14 6LT, UK. Email: smehmet@nhs.net

Br J Diabetes Vasc Dis 2015;15:40 http://dx.doi.org/10.15277/bjdvd.2014.030

Approximately 35 million people within the UK now use smartphones and they are an attractive option for delivering health interventions because of their technical capabilities and ease of use. It's logical that clinicians should be involved in the development of apps, people with diabetes may be more likely to use them if recommended or created by a trusted source.

Many diabetes apps are about tracking data such as blood glucose level, insulin dose, calories, weight, and often appear as mobile versions of existing paper records. Apps of this nature are a useful way of tracking one's health but lack educational content and don't necessarily support decision making or problem solving behaviour, which could potentially be achieved through using mobile technology. The 'Diabetes Key Tips' app was born out of a desire to improve information and support diabetes self-management and arrived to the Apple store on 30th March 2014.

The app interface was designed to resemble a pack of cards, and the user able to select which card to pick from a choice of objectives (Figure 1a).

Filters, on the 'About Me' screen, define the user in terms of gender, pregnancy plans, smoker/non-smoker, diabetes type 1 or 2, insulin treated and, if so, type of regime (Figure $1 b$ ).

Adding these filters ensures that the user has personalised information. Once an objective is chosen another screen is entered, and the user is able to enter numerical data for $\mathrm{HbA}_{1 c}$, pre-meal blood glucose level, BP, cholesterol or BMI. If the numerical value entered is outside of the target range the user is advised to make this an objective (Figure 1c), and enters a further screen listing a choice of topics to review (Figure 1d) with access to tips that

Figure 1. Smartphone screenshots

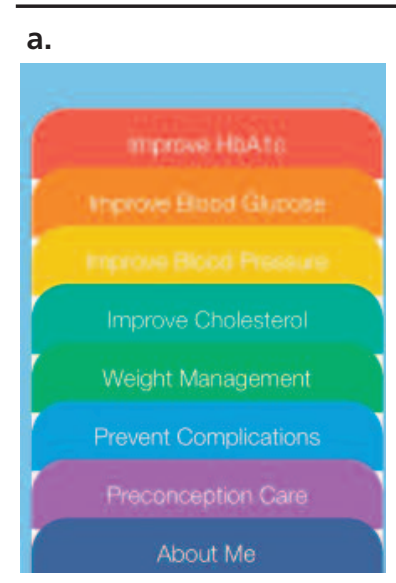

d.

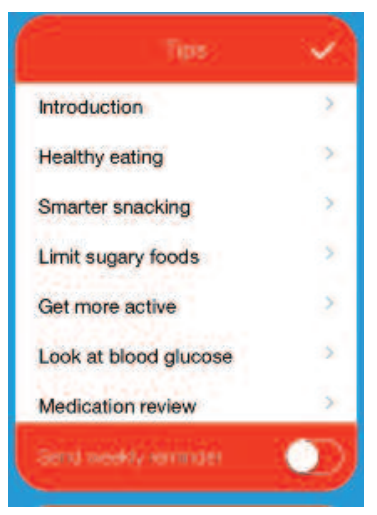

b.

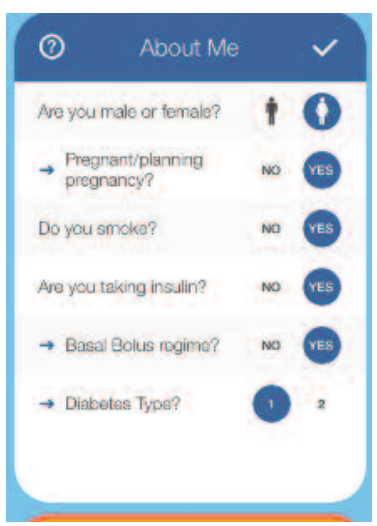

e. c.

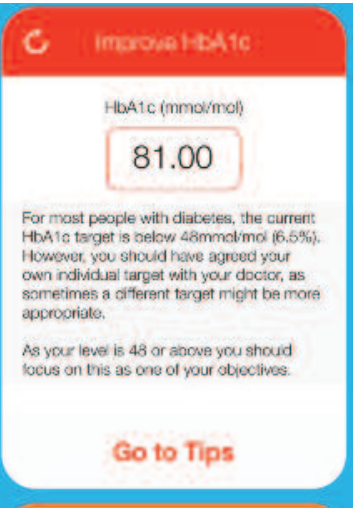

e.

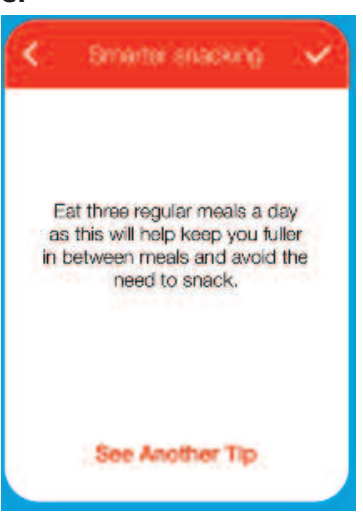

help to improve the relevant objective (Figure 1e).

\section{Summary}

The 'one size fits all' approach to managing people with diabetes doesn't always work, having a spectrum of available options and tools is important. The younger generation, many of whom are already technologically savvy, seem more likely to be responsive to mobile health technology.

Apps such as this support self-management by allowing users to set objectives, guides them to solve problems, provide tailored information and supports behaviour change. Feedback from healthcare professionals and people with diabetes will be valuable in helping to evolve the app further, and make it a tool that the diabetes community is willing to prescribe and use.

Conflict of interest Novo Nordisk provided a grant to support the production of the app, but Novo Nordisk has had no editorial control of the content. 